\title{
Synthesis of Combined Heat and Mass Exchange Networks Via a Trust Region Filter Optimisation Algorithm Including Detailed Unit Designs
}

\author{
Saif R. Kazi ${ }^{\mathrm{a}}$, Michael Short ${ }^{\mathrm{b}, *}$, Lorenz T. Biegler ${ }^{\mathrm{a}}$ \\ ${ }^{a}$ Carnegie Mellon University, 5000 Forbes Avenue, Pittsburgh 15213, USA \\ ${ }^{b}$ University of Surrey, Stag Hill, Guildford GU2 7XH, UK \\ m.short@surrey.ac.uk
}

\begin{abstract}
Mass and heat integration are important to achieving economically and environmentally sustainable processes through increased efficiency. Typically, heat and mass exchange networks are solved separately using process integration techniques such as pinch technology or formulating nonconvex mixed-integer nonlinear programming (MINLP) problems, which are challenging to solve. To simplify the MINLP, shortcut models are employed, which can result in under/overestimation of the real network, leading to suboptimal or infeasible designs. We introduce a new optimisation algorithm for combined heat and mass exchanger network synthesis (CHAMENS), including detailed design models. The method uses shortcut models in an MINLP to find network topology, followed by a nonlinear programming (NLP) suboptimisation. The NLP allows non-isothermal and non-isocompositional mixing, uses detailed unit models of packed columns based on orthogonal collocation on finite elements (OCFE), and detailed shell and tube heat exchanger designs. We incorporate a differential-algebraic equation (DAE) based shell and tube heat exchanger design model via surrogates in a trust region filter (TRF) framework, guaranteeing optimal solutions for the detailed exchanger models are found by the surrogate models. We demonstrate the proposed approach on a case study, showcasing its performance and the need to incorporate detailed unit models in topology optimisation to find practical optimal designs.
\end{abstract}

Keywords: heat exchanger networks, mass exchanger networks, optimisation, mixedinteger nonlinear programming, process integration.

\section{Introduction}

Mass and heat integration increase process efficiency by reducing external heating and cooling utilities and mass separating agents (MSAs), thus maximising heat and mass recovery within the process. To solve the problem using mathematical programming techniques, mass exchanger networks (MENs) and heat exchanger networks (HENs) are commonly formulated as separate MINLPs using superstructure-based approaches such as the stage-wise superstructure (SWS) for HENs proposed by Yee and Grossmann (1990), or the approach of El-Halwagi and Manousiouthakis (1990a) for MENs. These require nonconvex MINLP formulations, which are difficult to solve, and therefore unit representations are simplified, with heat exchanger area and mass exchanger height used as sizing parameters and heat and mass transfer coefficients fixed. Despite 
abundant literature on solving these problems separately, mass and heat transfer coefficients are related to temperatures and velocities and therefore there is a need to integrate these problems in combined heat and mass exchanger networks (CHAMENs). This is especially important in gas-liquid absorption, where there are benefits in operating absorption at low temperature and MSA regeneration at higher temperatures.

Srinivas and El-Halwagi (1994) formulated CHAMENS problems for the first time, using a two-step procedure, where an MINLP is first used to obtain the MEN with lowest MSA costs, followed by a HEN synthesis step. Liu et al. (2015) also solved the two networks separately using the SWS MINLP formulation to solve for network topologies before formulating an NLP to combine the models and optimise interactions between them. Recently, Kim et al. (2020) developed combined model for solving large MINLPs with MENs, HENs, and a newly developed regeneration exchanger network (REN), which incorporates different regeneration options. All these approaches, however, only use simplified representations for the unit operations and design models.

In MENS, without accounting for detailed aspects of unit designs, simplified formulations may result in sub-optimal or practically infeasible networks with flooding, low mass transfer coefficients or implausible L/D ratios. Isafiade and Short (2016) incorporated details of packed column unit design into the MINLP, such as column diameter, mass transfer coefficients, velocities, and noticed vastly different designs; however, this was difficult to converge due to the increased nonconvexity. Short et al. (2018) developed detailed packed column models based on discretisation of the differential equations to formulate a DAE model that can be solved via NLP. These models solve reliably, allowing for consideration of optimal diameters, heights, packing size, etc, while considering practical aspects such as column flooding. The authors then incorporated these into the MEN SWS model via a two-step optimisation algorithm, based on the work of Short et al. (2016), by using correction factors to ensure convergence of the MINLP shortcut models to the solutions of the detailed designs.

Recently, Kazi et al. (2020a) developed a shell and tube heat exchanger design optimisation model that uses enumeration to determine discrete decisions on baffles, number of shells, tube passes, fluid allocation, etc., followed by a detailed DAE model. These models were incorporated into HENS, allowing for designs to incorporate detailed exchangers, including pressure drops, multiple shells, etc. (Kazi et al., 2020b). More recently, Kazi et al. (2020c) developed a novel algorithm for including these models into HENS as surrogates via a TRF algorithm from Yoshio and Biegler (2020).

In this work, we propose a two-stage approach where, in the first stage, an MINLP superstructure-based optimisation is used with simplified, shortcut unit models to find topologies for the CHAMEN. Next, an NLP is formulated with detailed optimisation unit models for the packed bed absorption columns, based on OCFE (Short et al., 2018). These models are directly incorporated in the NLP. Detailed shell-and-tube heat exchanger design models, from Kazi et al. (2020a), are incorporated into the flowsheet via a TRF algorithm. The algorithm allows for optimisation of a detailed flowsheet with first-principles models, and with superstructure decisions decided via a simultaneous optimisation procedure. The method does not increase the complexity of traditional MINLP process integration models, while providing a method of incorporating detailed unit designs. The algorithm and its application to CHAMENs demonstrates the power of the approach, highlighting the need for detailed unit models in superstructure-based process synthesis, and proposes a potential framework for future process synthesis. 


\section{Modelling framework}

\subsection{Topology optimisation}

We present a modelling framework for CHAMENS that begins with solving the MEN, HEN, and REN separately as MINLPs to obtain initialisations for the full problem. Note that these formulations are very similar to common SWS approaches mentioned earlier, with a REN model following similar formulation to Kim et al. (2021). These models assume constant heat and mass transfer coefficients, isothermal and isocompositional mixing, and use shortcut formulations to represent units. By doing this, we can maintain the model formulation as mostly convex and reduce complexity for the large MINLP formulation, which is challenging to solve. Following this, the problems are integrated through linking variables, with temperatures and flowrates of MSAs. Parameters are selected to ensure that the MINLP problem underestimates the objective function, thus ensuring that this can act as a lower bound to the overall minimisation problem. If a global solution is found to the MINLP problem, this is a rigorous lower bound. However, in practice this cannot be guaranteed due to non-convexity of the formulation.

\subsection{Detailed network optimisation}

The topology obtained from the shortcut models is used to form an NLP, consisting of 3 linked submodels, solved simultaneously, for the REN, MEN, and HEN. The MEN and REN are formulated using the NLP optimisation model proposed by Short et al. (2018), where each selected mass exchanger is solved as a DAE model of a packed column using OCFE. In Short et al. (2018), the mass balances over each column were fixed to that of the solution from the MINLP, however in our formulation, we allow for inlet and outlet concentrations of lean and rich streams to be optimised, along with column designs. Furthermore, the model allows non-isocompositional mixing of split streams and temperature-dependent mass transfer coefficients, linked via the Henry's Law coefficient. The MEN and REN submodels are connected via the variable flowrates for the MSAs that are regenerated. The HEN NLP consists of the energy balance equations from the MINLP, with fixed binary variables and nonisothermal mixing. Shell and tube heat exchanger design variables that affect the overall objective function, such as number of shells, pressure drops, and areas, are included via reduced order models (ROMs). These ROMs are updated at each iteration of the TRF algorithm to ensure that they are accurate within a small trust region around the previous solution, obtained from the running the detailed discretised DAE model of Kazi et al. (2020a). For more details on the TRF strategy, see the work of Yoshio and Biegler (2020). The NLP is solved at each iteration of the TRF, including all NLP subproblems, with the HEN NLP linked to the MEN and REN NLPs via the temperatures and flowrates of the lean streams in the MEN, which are also the flowrates of the rich streams in the REN.

\subsection{Integer-cut algorithm and implementation}

Once the NLP TRF algorithm terminates, the solution includes optimal flowrates of utilities and detailed designs of individual units. These designs include optimal pressure drops, tube and shell passes, areas, fluid allocation and tube lengths for the shell and tube heat exchangers, and the optimal packing size, column heights, diameters, velocities, and stream splits for the packed columns in the MEN and REN. Additionally, the optimal temperatures for mass exchange are also obtained. This solution acts as an upper bound to the overall algorithm. Integer cuts can then be generated to exclude the 
previous topology from future iterations of the MINLP, allowing other topologies to be evaluated. Once the lower bound exceeds the upper bound, the algorithm terminates, with the upper bound solution as optimal. A graphic depiction of the algorithm is shown in Figure 1. The full strategy is implemented in Python, using Pyomo (Hart et al., 2017), with NLP problems being solved with IPOPT 3.13.3 (Waechter and Biegler, 2006) and MINLP problems solved using BARON (Tawarmalani and Sahinidis, 2005). Note that due to the size and nonconvexity of the MINLP, on certain iterations it may be necessary to relax tolerances in BARON to find feasible topologies for next iterations.

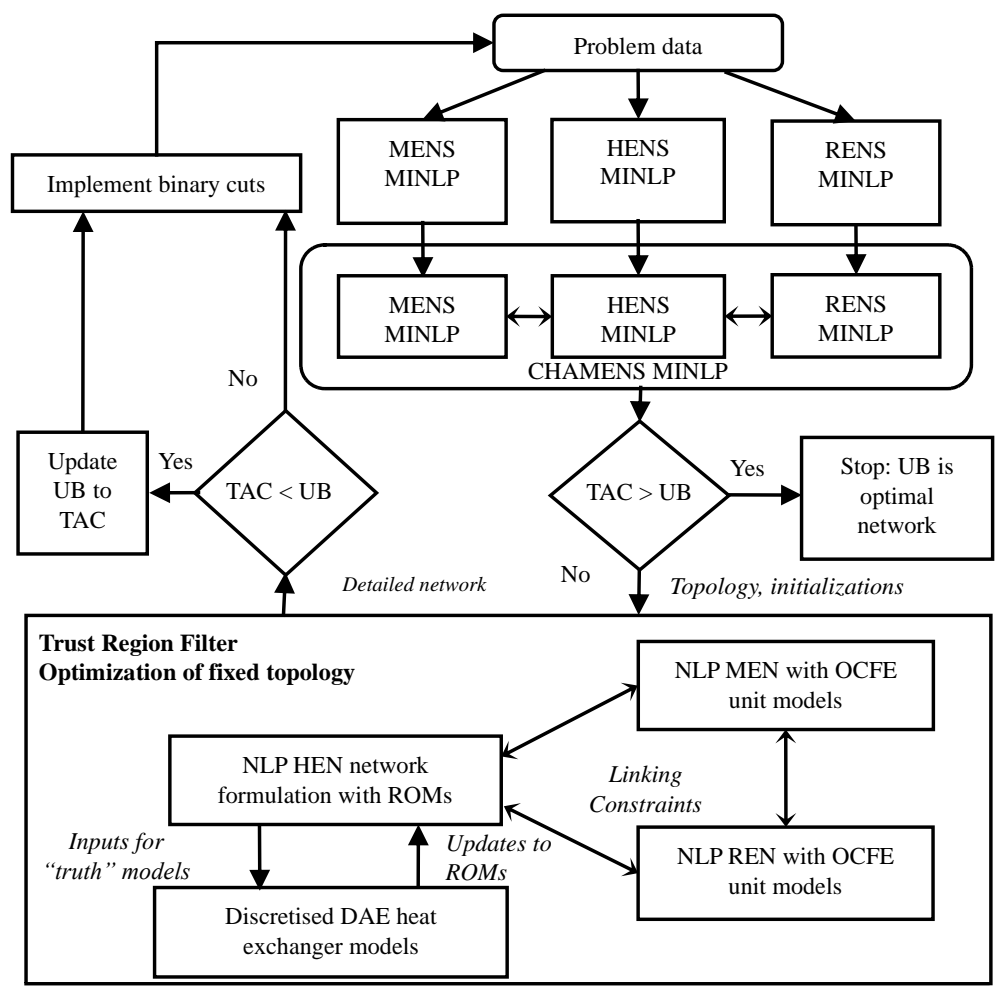

Figure 1: Overall solution algorithm

\section{Case Study}

We formulate an $\mathrm{H}_{2} \mathrm{~S}$ removal process, where coke-oven gas (COG) is sweetened. The problem has two rich streams, $C O G\left(R_{1}\right)$ and tail-gas from a Claus unit $\left(\mathrm{R}_{2}\right)$, both rich in $\mathrm{H}_{2} \mathrm{~S}$, potentially absorbed by three lean streams: aqueous ammonia $\left(\mathrm{L}_{1}\right)$, a process MSA, and external MSAs $\left(\mathrm{L}_{2}\right) 15 \mathrm{wt} \%$ methyl diethanolamine (MDEA) and $\left(\mathrm{L}_{3}\right)$ chilled methanol regenerated via low pressure or medium pressure steam. For brevity, detailed problem data and model formulations are omitted, with results shown to demonstrate the algorithm. We use stream parameters from Kim et al. (2020), with flowrates multiplied by 10 and model formulations from aforementioned studies. For the purposes of this example we consider the temperature of $\mathrm{L}_{2}$ and flowrates of $\mathrm{L}_{2}$ and $\mathrm{L}_{3}$ as linking variables. The optimal design is shown in Figure 2. The MINLP consists of 460 constraints, 455 continuous variables and 64 discrete variables (TAC: \$26,209,870), and 
the NLP model consists of 11,199 constraints and 11,095 continuous variables (TAC: $\$ 26,634,598)$. The full algorithm solves in 5 CPU min, with most computational effort spent in the TRF algorithm. Interestingly, the result finds that $\mathrm{L}_{2}$ and low-pressure steam are not needed in the optimal network. Note that there are significant discrepancies in the area of the heat exchanger and column designs between the NLP and MINLP solution, showing the need for detailed designs. Interesting differences at this point are that each column is constrained by flooding allowance, with the velocities and packing sizes selected to ensure that each column is operating at high mass transfer. This generally results in cheaper columns than the initial MINLP shortcut models in this system, as diameters are available for optimisation. Interestingly, the NLP also redistributes mass load between the first 2 exchangers on $\mathrm{R} 1$ in a different way to the MINLP, resulting in a slight decrease in methanol use and subsequent reduced costs.

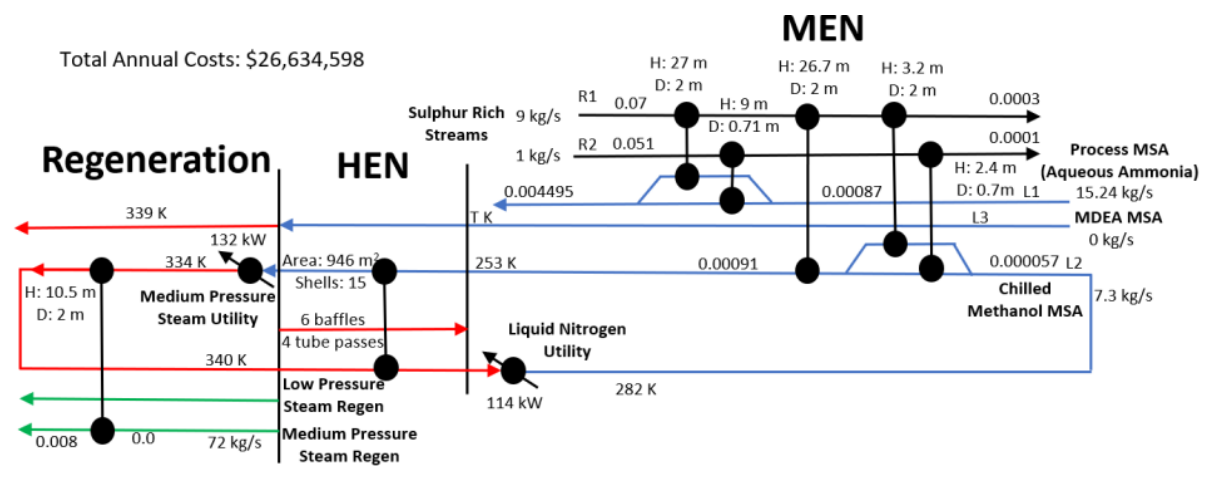

Figure 2: Optimal network identified for the Case Study

The shell and tube heat exchanger design requires many shells and high pressure drop and the NLP optimisation attempts to reduce the area of this exchanger, particularly since, in the NLP, a fixed cost is introduced for numbers of shells, and pressure drop is also added into the objective function. On the second iteration of the MINLP, following the integer cut, only utilities (and no process-process heat exchangers) are selected and the new TAC is significantly worse due to high cooling costs.

\section{Conclusions}

We develop a new algorithm for solving CHAMENS that is the first to include detailed unit models as part of the optimisation formulation. The algorithm uses an MINLP model formulation with shortcut models to represent units to solve the large, nonconvex combinatorial problem first with parameters chosen to ensure an underestimation of the objective function. This solution serves as the topology for the subsequent algorithmic steps. With topology fixed, an NLP model is then formulated. The NLP includes detailed mass exchanger designs, non-isothermal/non-isocompositional mixing constraints, and the mass and energy balances. Shell and tube heat exchangers are incorporated via surrogate models, generated from a detailed heat exchanger design algorithm, within a TRF optimisation algorithm, which guarantees that optimal solutions for the detailed exchanger models are found by the surrogate models. We demonstrate the technique on a case study involving the removal of $\mathrm{H}_{2} \mathrm{~S}$ from gaseous waste streams and find interesting designs that are different from those obtained using MINLPs alone. We present detailed heat exchanger designs and packed column designs, 
solving the large NLP quickly to show that the approach can be easily extended. Future work will focus on developing detailed regeneration models and including different column types that are common in MENs, in addition to a planned release of the code as an open-source heat and mass integration package. The flexible approach may also be modified to multi-component and reactive MENs in future work.

\section{References}

El-Halwagi M.M., Manousiouthakis V., 1990a, Automatic synthesis of mass exchange networks with single component targets. Chem. Eng. Sci., 45(9), 2813-2831.

Hart W.E., Laird C.D., Watson J.-P., Woodruff D.L., Hackebeil G.A., Nicholson B.L., Siirola J.D., 2017, Pyomo - Optimization Modeling in Python, Springer, Cham, Switzerland.

Liu L., Du J., Yang F., 2015, Combined mass and heat exchange network synthesis based on stage-wise superstructure model, Chinese Journal of Chemical Engineering, 23, 1502-1508

S.R. Kazi, M. Short, L.T. Biegler, 2020a, Heat Exchanger Network Synthesis With Detailed Exchanger Designs - 1. A Discretized Differential Algebraic Equation (DAE) Model for Shell and Tube Heat Exchanger Design, AIChE J., e17056.

S.R. Kazi, M. Short, L.T. Biegler, 2020b, Heat Exchanger Network Synthesis With Detailed Exchanger Designs - 2. Hybrid Optimization Strategy for Synthesis of Heat Exchanger Networks with Detailed Individual Heat Exchanger Designs, AIChE J., e17057.

S.R. Kazi, M. Short, L.T. Biegler, 2020c, Heat Exchanger Network Optimization including Detailed Heat Exchanger Models using Trust Region Method, Comp. Aid. Chem. Eng., 48, 1051-1056.

S-M. Kim, A.J. Isafiade, M. Short, 2021, The synthesis of combined heat and mass exchange networks (CHAMENs) with renewables considering environmental impact. Recent Advances in Process Intensification and Integration for Sustainable Design, Wiley-VCH.

M. Short, A.J. Isafiade, D.M. Fraser, Z. Kravanja, 2016, Synthesis of heat exchanger networks using mathematical programming and heuristics in a two-step optimisation procedure with detailed exchanger design. Chem. Eng. Sci., 144, 372-385.

M. Short, A.J. Isafiade, L.T. Biegler, Z. Kravanja, 2018, Synthesis of mass exchanger networks in a two-step hybrid optimization strategy. Chem. Eng. Sci., 178, 118-135.

A.J. Isafiade, M. Short, 2016, Synthesis of mass exchange networks for single and multiple periods of operations considering detailed cost functions and column performance. Proc. Safety and Env. Prot., 103, 391-404.

B. K. Srinivas and M. M. El-Halwagi, 1994, "Synthesis of combined heat and reactive massexchange networks,” Chem. Eng. Sci., 49, 2059-2074.

Tawarmalani M., Sahinidis N.V., 2005, A polyhedral branch-and-cut approach to global optimization, Math. Program., 103(2), 225-249.

Wächter A., Biegler L., 2006, On the implementation of an interior-point filter line-search algorithm for large-scale nonlinear programming, Math. Program., 106, 25-57.

T.F. Yee, I.E. Grossmann, 1990, Simultaneous Optimization Models for Heat Integration - II. Heat Exchanger Network Synthesis, Comp. \& Chem. Eng., 14, 1165-1184.

N. Yoshio, L.T. Biegler, 2020, Demand-based optimization of a chlorobenzene process with high fidelity and surrogate reactor models under trust region strategies, AIChE J., 17054. 